\title{
REVISIÓN SISTEMÁTICA: SITUACIÓN ACTUAL DE LA PERSONALIDAD DE MARCA PARA EL POSICIONAMIENTO ESTRATÉGICO
}

\author{
SYSTEMATIC REVIEW: CURRENT STATUS OF BRAND PERSONALITY FOR \\ STRATEGIC POSITIONING
}

\section{REVISÃO SISTEMÁTICA: SITUAÇÃO ATUAL DA PERSONALIDADE DA MARCA PARA POSICIONAMENTO ESTRATÉGICO}

Jose Joel Cruz Tarrillo; Karla Liliana Haro Zea; Juan Jesús Soria Quijaite

\begin{abstract}
Magíster en Administración de Negocios con Mención en Gestión Empresarial, Universidad Peruana Unión. Coordinador de Investigación en la Escuela Profesional de Administración, Universidad Peruana Unión. ORCiD: 0000-0002-6372-5055. E-mail: jose.cruz@upeu.edu.pe, Perú.
\end{abstract}

Doctora en Planeación estratégica y Dirección de Tecnología por la Universidad Popular Autónoma del Estado de Puebla (UPAEP) con Postdoctorado en Desarrollo Sostenible. Profesora investigadora de la Universidad de Montemorelos. ORCiD: 0000-0002-7257-0673. E-mail: liliana.haro@outlook.com, México.

Doctor en Ingeniería de Sistemas, Universidad Alas Peruanas. Docente de la Escuela Profesional de Ingeniería de Sistemas, Universidad Peruana Unión. Investigador RENACYT, Grupo de María Rostworowski del Nivel I. ORCiD: 0000-0002-4415-8622. E-mail: jesussoria@ upeu.edu.pe, Perú. 


\title{
Resumen
}

El propósito de esta investigación es exponer el avance de la personalidad de marca (PM) para fines de posicionamiento estratégico $(\mathrm{PE})$ cuyo análisis involucre herramientas, modelos y sectores económicos. La metodología utilizada es una revisión sistemática de la literatura con una ventana de observación de 2018 a 2021; los principales hallazgos muestran que el análisis de correspondencia y el mapeo perceptual son herramientas que permiten medir el PE. Las principales contribuciones fueron en el campo del turismo, deporte, educación, automotriz, mercado internacional, política, retail, comercio electrónico, productos de aseo personal, servicios portuarios, publicidad, bebidas y periodismo. Los modelos que han surgido son el BCP, modelo para deportistas y el modelo STPS que permiten mejorar el PE desde la teoría de la PM, así como propuestas innovadoras mediante el uso de nuevas herramientas como emojis, índices, cuadro de divergencia y minería de textos.

Palabras clave: estrategias de comunicación; imagen de la marca; marketing; personalidad de marca; posicionamiento estratégico.

JEL: M1; M3; M31; M37; M39

\begin{abstract}
The purpose of this research is to expose the advancement of the brand personality (BP) for strategic positioning purposes (SP) whose analysis involves tools, models, and economic sectors. The methodology used is a systematic review of the literature with an observation window from 2018 to 2021; the main findings show that correspondence analysis and perceptual mapping are tools that allow measuring SP. The main contributions were in the field of tourism, sports, education, automotive, international market, politics, retail, electronic commerce, personal hygiene products, port services, advertising, beverages, and journalism. The models that have emerged are the BCP, a model for athletes and the STPS model that allow improving the SP from the theory of BP, as well as innovative proposals using new tools such as emojis, indices, divergence table and text mining.
\end{abstract}

Keywords: communication strategies; brand image; marketing; brand personality, strategic positioning.

JEL: M1; M3; M31; M37; M39 


\section{Resumo}

O objetivo desta pesquisa é expor o avanço da personalidade de marca (PM) para fins de posicionamento estratégico (PE), cuja análise envolve ferramentas, modelos e setores econômicos. A metodologia utilizada é uma revisão sistemática da literatura com janela de observação de 2018 a 2021; Os principais achados mostram que a análise de correspondência e o mapeamento perceptivo são ferramentas que permitem mensurar o PE. As principais contribuições foram nas áreas de turismo, esportes, educação, automotivo, mercado internacional, política, varejo, comércio eletrônico, produtos de higiene pessoal, serviços portuários, publicidade, bebidas e jornalismo. Os modelos que surgiram são o BCP, um modelo para atletas e o modelo STPS que permitem aprimorar o PE a partir da teoria do PM, bem como propostas inovadoras por meio da utilização de novas ferramentas como emojis, índices, tabela de divergência e text mining.

Palavras-chave: estratégias de comunicação; imagem de marca; marketing; personalidade da marca, posicionamento estratégico.

JEL: M1; M3; M31; M37; M39

\section{Introducción}

En el contexto en que nos desarrollamos donde los cambios son impredecibles, las empresas tienen que crear y mantener una posición competitiva, a fin de soportar los constantes cambios y la competencia (Escobar et al., 2017). En los últimos años el consumidor ha tomado un papel preponderante en la empresa, es por ello que dirigirse de forma adecuada determina el éxito de la empresa (Glińska \& Rudolf, 2019); en ese sentido las marcas deben proyectar personalidades que sean congruentes con la de los clientes (Lara et al., 2019); para establecer fuertes conexiones emocionales hacia la marca (Ramos, 2019).

La marca se define como una señal, nombre, diseño, símbolo, que identifica y diferencia un producto o servicio de los competidores (Asociación Americana de Marketing, 2019; Escobar et al., 2017; Kotler et al., 2017). Su propósito es añadir valor a los productos generando características únicas que le permitan ser fuertes en mercado (Baştuğ et al., 2019). 
La personalidad de marca (PM) tiene sus orígenes a partir del estudio de Foxall \& Haskins (1986) , debido a la necesidad de conocer la conducta de los consumidores (Shank \& Langmeyer, 1994). Sin embargo, el debate de PM se origina en la década de los noventa (Garanti \& Kissi, 2019; Bennett, 1997). Se indicaba que una marca en el contexto empresarial tiene similitud con la personalidad de un individuo (Van, 1997). Sin embargo, marcaría la pauta el estudio de Aaker (1997) en la cual hace una aproximación conceptual en la que indica es el conjunto de características humanas asociadas a la marca, representan valores y características que pueden ser percibidas como jóvenes, cercanos, modernos y simpáticos jugando un rol importante en la congruencia con el cliente (Liu et al., 2019).

Al respecto Siguaw et al. (1999) conceptualizan la PM como la agrupación de características o rasgos humanos que los clientes señalan de una marca. Como consecuencia del modelo propuesto por Aaker (1997) han surgido diversos estudios en la primera década de este milenio, básicamente se han enfocado a replicar en diferentes ámbitos económicos, tales como textilería (Kim, 2000); cultura (Aaker et al., 2020); gastronomía (Murase \& Bojanic, 2004); automóvil (Rojas et al., 2004) y turismo (Hosany et al., 2006; Ekinci y Hosany, 2006).

La PM ha tenido mucho interés por parte de los investigadores dado al dinamismo que enfrentan los sectores económicos, se requiere una constancia en las estrategias que puedan contribuir a mejorar el posicionamiento de la empresa. Es por ello la importancia de conocer los avances en la literatura respecto a la PM y el posicionamiento estratégico (PE). A continuación, se expone el avance de la PM para fines de PE, posteriormente se explica la metodología realizada, los resultados y conclusiones.

\section{Metodología}

\section{Definición de las preguntas de interés}

Esta revisión sistemática se interesa por explorar ¿Cuáles son las técnicas que han sido utilizadas de la PM para fines de PE? ¿Cuáles son los sectores económicos que han abordado la PM de cara al PE? ¿Cuáles son los modelos de PM para el PE? 


\section{Selección de la palabra clave}

Para la selección de los autores, los términos ingresados a la búsqueda fueron: Personalidad de marca (brand personality) y Posicionamiento Estratégico (Strategic Positioning), con una metodología de revisión sistemática de la literatura de acuerdo a Chicaíza et al. (2017). Es menester indicar que se hicieron diversas combinaciones a fin de identificar la más adecuada posible, esto se realizó con la ayuda de operadores booleanos.

\section{Selección de recursos}

Para tener un número significativo de recursos científicos, se realizó la búsqueda en las dos bases de datos de mucha relevancia en el ámbito académico científico, estas fueron Scopus $®$, Web of Science y Emerald, como se muestra en la Figura 1. En primera instancia se hizo la búsqueda en la base de datos Scopus ${ }^{\circledR}$ identificando a 34 artículos registrados, luego se hizo la búsqueda en la Web of Science encontrándose 78 artículos registrados y Emerald con 103. La exclusión de los artículos se realizó en base a los títulos, manuscritos completos, por encontrarse el mismo artículo en más de una base de datos y por no estar en la línea de esta investigación (Espinoza \& Gallegos, 2019).

\section{Figura 1}

Proceso de selección de los artículos
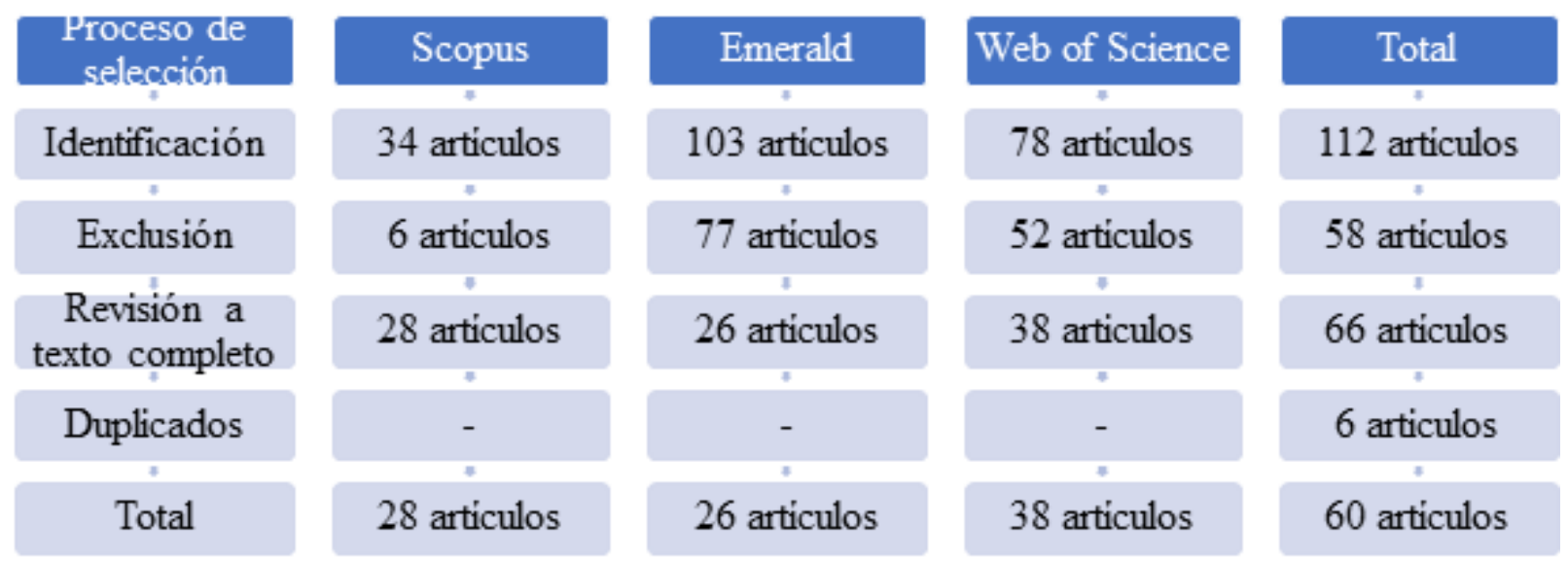

Nota: La selección estuvo compuesta por 60 artículos de 2018 al 2021.

Fuente: elaboración propia. 


\section{Construcción de ecuaciones de búsqueda}

La ecuación de búsqueda en la base de datos Scopus:

(TITLE-ABS-KEY (brand AND personality) AND TITLE-ABS-KEY (brand AND positioning)) AND (LIMIT-TO (PUBYEAR, 2021) (LIMIT-TO (PUBYEAR, 2020) OR LIMIT-TO (PUBYEAR, 2019) OR LIMIT-TO (PUBYEAR, 2018))

Ecuación de búsqueda en la base de datos Web of Science:

TOPIC: (brand personality) AND TOPIC: (Brand positioning). Refined by: DOCUMENT TYPES: (ARTICLE) Timespan: 2018-2021. Indexes: SCI-EXPANDED, SSCI, A\&HCI, ESCI.

Ecuación de búsqueda en la base de datos en Emerald:

(content-type:article) AND (abstract:"brand personality" AND (Strategic Positioning))

\section{Resultados}

En la Figura 2 se muestran los artículos analizados de acuerdo al año que fueron publicados. En ellos se encuentran técnicas, sectores económicos, y modelos de PM para fines de PE. Es importante notar que Estados Unidos de América ha sido el país que presenta la producción más alta relativa a la PM basados en el h-index y los autores con el mayor número de artículos son Yongjun Sung, Jennifer Aaker, Ulrich R. Orth, Michael Bosnjak, Gary Davies y Luiz Moutinho,

siendo Susan Fournier y Jennifer Aaker las autoras más influyentes (Llanos \& Merigo, 2019), con más de 12,000 citas en Google Scholar cada una solo relativas a las marcas, seguidas de Bernd Schmitt, con más de 7000 citas. 


\section{Figura 2}

Artículos analizados por año de publicación.

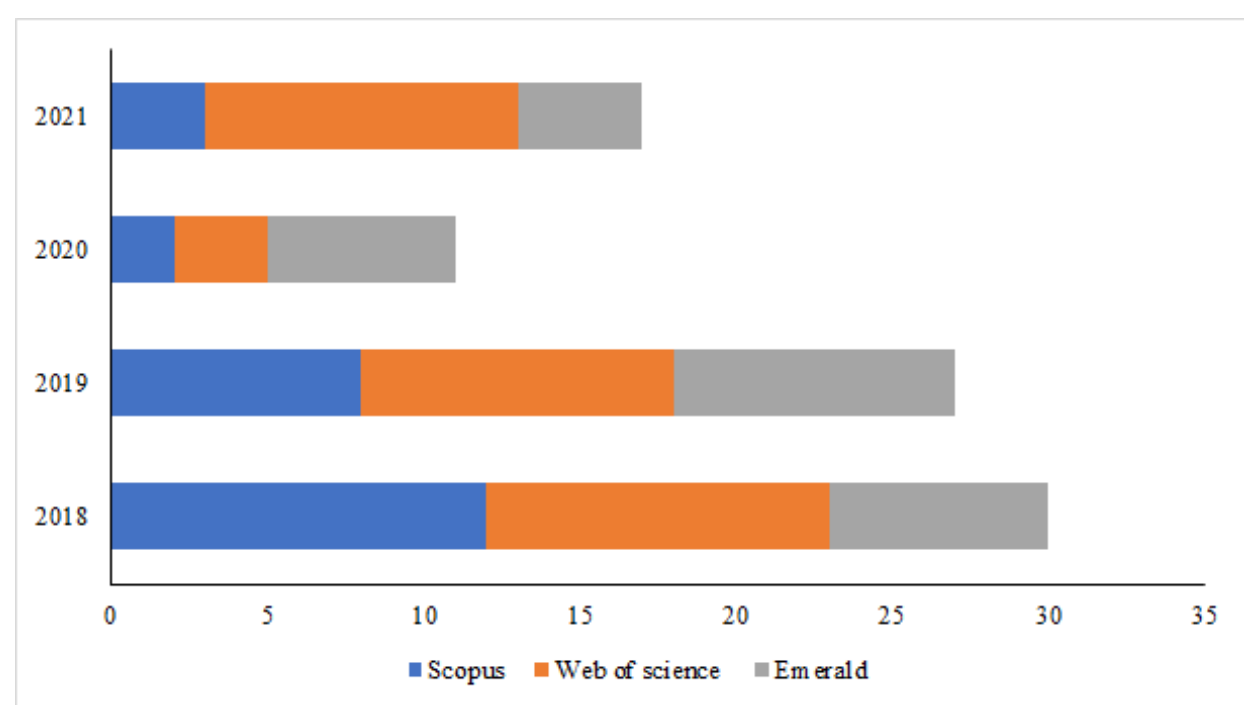

Fuente: elaboración propia.

\section{Personalidad de Marca}

El concepto de la PM ha sido usado desde 1950 (Ogilvy, 1955). Sin embargo, su conceptualización en el ámbito académico se materializó hasta que la autora Jennifer Aaker propuso una definición de la marca constructo de personalidad y sus dimensiones en 1997, estableciendo la base académica para su medición. De acuerdo con la Asociación Americana de Marketing (2021, p. 1), "La personalidad de la marca se refiere a la naturaleza psicológica de una marca en particular según la intención de sus vendedores, aunque las personas en el mercado pueden ver la marca de otra manera"; es decir, el concepto que toma como modelo la personalidad humana y lo asocia a las cualidades humanas, deseos y perspectivas. Para Aaker (1997, p. 348) 348 y Fournier (1998, pp. 344-345), los rasgos de personalidad de una marca se infieren de varias acciones de marketing, incluidos estilos publicitarios (es decir, patrocinador de celebridades, personaje, jingle, eslogan), la marca en sí (es decir, nombre, logotipo, símbolo), políticas de precios (es decir, skimming o penetración), y canales de distribución (es decir, personal de la tienda o de ventas). Cabe mencionar que la investigación de la PM inició una tendencia de crecimiento sostenido a la fecha, sin embargo, algunos autores consideran que no es simple poner en práctica la metáfora de la PM (Capelli \& Jolibert, 2009; Caprara, Barbaranelli \& Guido, 2001), otros afirman que existen problemas de 
generalización con el constructo en el proceso de validez. (Caruana et. al., 2007; Colmenares \& Saavedra, 2008).

Para Llanos \& Merigo (2019), "la PM muestra potencial importante para desarrollarse en dos áreas: la consolidación sistemática de la personalidad de la marca con la comunicación digital y el establecimiento de relaciones con otros conceptos fundamentales de marketing", asimismo, el artículo de JBM de Radler (2018) mediante una revisión bibliométrica de 20 años de literatura identificó cinco corrientes de investigación denominadas: efectos directos e indirectos de PM; dinámica de las dimensiones de la PM; PM en extensiones de marca; aplicación de PM a áreas relacionadas; y medición de la PM, en suma, Moussa (2021), midió la PE mediante un lenguaje visual-emoji, mientras que la presente investigación se sitúa en exponer las técnicas utilizadas, modelos y sectores económicos que han estudiado la PE mediante la revisión sistemática de literatura de los años 2018-2021.

\section{Posicionamiento estratégico}

La competitividad determina el éxito o fracaso de las empresas y la estrategia coadyuva en la búsqueda de una posición favorable dentro de la industria (Porter, 2016). En este sentido un elemento central de la estrategia es el PE de la marca, por lo que, "el valor de las marcas alcanzaron en las negociaciones de fusiones y adquisiciones entre grandes compañías que son en realidad, adquisiciones de posicionamientos en las mentes de los clientes potenciales, con sus conjuntos de asociaciones, calidades y diferencias" (Kapferer, 1992, p. 16), sin embargo, para Jaksic et al., (2019), la efectividad de las actividades de gestión de marca dependerá significativamente sobre los detalles de sus características comercialmente explotables, y "su posicionamiento adquiere una mayor importancia en función de la realidad del mercado" (Aaker, 1996, p. 11), por lo que de acuerdo con Hooley \& Saunders (1996) la llave para el éxito está en la implementación eficaz del concepto de marketing a través de estrategias de posicionamiento, así entonces es preciso notar que existen diferentes aportaciones al concepto; para ello, Kotler (2000, p. 270) dice que "el posicionamiento es el acto de desarrollar la oferta y la imagen de la empresa, de forma que ocupen un lugar distinto y valorado en las mentes de los consumidores objetivo, y Gouvêa (1991) resalta el tenor estratégico implícito en la técnica de posicionamiento, que consiste en el mapa de rutas que se trazarían para determinar cómo ir del punto A al punto B. Adicionalmente, es importante 
diferenciar dos conceptos de posicionamiento: El PE, tipificado en el trabajo de Porter (1989; 2016), que designa el lugar que una empresa ocupa en su industria a partir de una estrategia competitiva y, el posicionamiento operacional definido por Ries \& Trout (2002, p. 2) como el modo en que usted se diferencia en la mente de su cliente potencial. En esta línea, Alcaniz \& López (2000) plantean la orientación al cliente basada en los esquemas perceptuales, y la orientación a lo organizacional, centrada en el posicionamiento de la compañía en la industria, mientras que para Serralvo \& Tadeu (2005), es más adecuado considerar un posicionamiento perceptual y organizacional, donde ambos tienen implicaciones de carácter estratégico y pueden extenderse a programas de largo plazo.

En palabras de Kotler \& Keller (2012, p. 299), el posicionamiento de una marca atractiva y bien diferenciada requiere un profundo conocimiento de las necesidades y deseos del consumidor, así como de las capacidades de la empresa y de las acciones de la competencia. Sin duda, vivimos en tiempos de constante cambios, por lo que las empresas deberán identificar sus oportunidades clave y reconsiderar el posicionamiento estratégico de su marca.

\section{Técnica de personalidad de marca para lograr el posicionamiento estratégico}

La lucha de las marcas por mantenerse siempre vigentes, atractivas y deseables por los consumidores ha permitido que en estos últimos años surjan técnicas tales como el mapeo perceptual y análisis de correspondencia que permiten un PE.

El mapa perceptual, es una de las técnicas usadas ampliamente en el marketing cuya función es analizar la composición del mercado, diseñar nuevos productos, mide la posición de las marcas ( Rasooli et al., 2018; Hauser \& Koppelman, 1979). Su propósito es reflejar la percepción de los clientes respecto a la marca, el producto, los servicios, competencia, identifica el estado de cada marca de acuerdo a los atributos estudiados (Steenkamp et al., 1994; Chacko et al., 2019).

Tradicionalmente esta técnica ha sido usada en su nivel descriptivo, sin embargo, en estos últimos años ha trascendido usándose en el nivel inferencial (Iacobucci \& Grisaffe, 2018; Choudhury et al., 2019). Además, se ha usado la web como fuente de datos para recopilar percepciones, deseos, 
sugerencias, comentarios de los consumidores (Won et al., 2018; Vriens et al., 2019; Ruei et al., 2019).

El análisis de correspondencia, por su versatilidad puede ser aplicado en diversos escenarios y sectores económicos (Hoare \& Bock, 2019). Además, permite medir relaciones y diferencias entre marcas Baştuğ et al. (2019). En este orden de ideas Rutter et al. (2019) el análisis de correspondencia están involucrados varias categorías y que se puede reducir a un eje bidimensional para una mejor interpretación visual. Es así, que Hanna y Rowley (2019) usan para investigar el posicionamiento que genera la PM de las capitales europeas y Rutter et al. (2018) la utiliza en los partidos políticos.

\section{Personalidad de marca para fines de posicionamiento estratégico}

En el mundo actual establecer el nombre de una marca siempre es un aspecto fundamental para fines de imagen y su posicionamiento en la mente del consumidor. El nombre ideal para una marca es difícil encontrarlo Gita et al. (2018). Sin embargo, se vuelve más difícil aún lograr una relación con la cultura de las sociedades y personalidad de los consumidores.

Existen diversas contribuciones de PM para fines de PE de acuerdo al sector económico, como se muestra en la Figura 3. El sector donde mayores estudios se han realizado en estos últimos años es el turismo, luego el deportivo, educación, automotriz, mercado internacional, política, retail, comercio electrónico, productos de aseo personal, servicios portuarios, publicidad, bebidas y periodismo, las cuales se analizan a continuación.

El turismo, es un sector brinda aportes sustanciales a la economía de cada nación, es por ello que se hace esfuerzos denodados para lograr un PE adecuado. Un destino turístico es una marca, en ese sentido pueden convertirse en hedónicos, es decir las que son gratificantes o utilitarios que satisfacen necesidades primarias Deb (2020). Esto dependerá de la estrategia PE y la personalidad que se pretenda proyector agnusson et al. (2019), a fin de establecer asociaciones positivas en la mente de los consumidores Rutter et al. (2019). 
La utilización a los llamados influencers para posicionar las marcas, es indispensable que exista congruencia entre la personalidad del influencers y PM (Kim et al., 2018). De acuerdo con la teoría de esquemas de desarrollo Schlansker (1946) la congruencia entre PE y PM del destino turístico hacen que los consumidores se interesan y sea un factor clave de decisor de para elegir el destino. En el mundo existen ciudades muy populares, posicionadas como destinos turísticos Glińska \& Rudolf (2019) esto se logra, proyectando una personalidad que genere atracción centrarse no solo en el turista potencial, sino que debe ser aceptada por la comunidad, compartiendo una visión común entre las partes interesadas (Baćac \& Demonja, 2021). Sin embargo, los esfuerzos denodados por convertir a los destinos turísticos en el más popular para los visitantes se han visto obligados a cerrar por la enfermedad del coronavirus causada por el COVID 19. Actualmente, existe una necesidad de una comunicación efectiva con respecto al cumplimiento de los protocolos de salubridad.

El modelo de PM de Aaker (1997) compuesto por cinco categorías (sinceridad, emoción, competencia, sofisticación, rudeza), ha sido muy usado en este sector, destacándose los rasgos de amigable, actualizada, único, confiable, exitoso y emocionante como los más importantes (Lee \& Kim, 2018; Rutter et al.,2018; Su \& Reynolds, 2019).

Ahora bien, resulta un desafío construir una marca fuerte y consistente debido a que existen diversos segmentos turísticos al que se tiene que atender Vinyals y Mohammadi (2018) y cada segmento muestran comportamientos distintos Quintal et al. (2019), se hace necesario identificar los rasgos de PM más relevantes que identifican a este segmento para generar felicidad en los consumidores Rai \& Kumar (2018).

Las investigaciones también se han desarrollado en el ámbito del periodismo, básicamente para evaluar la PM e impulsar la marca personal de los hombres de prensa López et al. (2020). Las estrategias que se implementen para posicionarse deben ser distintos a su competencia, destacar su ventaja a fin de ser el centro de la atención (Aaker, 2020). Por otro lado, existe una preocupación por parte los Mercadólogos al momento de intentar posicionar una marca desfavorecida Nazim \& Rajeswari ( 2019) puesto que por aspectos de presupuesto y lo difícil de hacer conocido algo no 
existe para otros. En ese sentido se recomienda usar el efecto underdog a fin de consolidar una marca desfavorecida.

Vivimos en un mundo completamente globalizado, en la que cliente están por diferentes países. Además de la PM, la calidad, la imagen, la reputación y la cultura son factores clave para lograr un PE adecuado (Habibi et al.,2018; Kim et al., 2018; Rojas et al.,2018) hacen una crítica al modelo de Aaker (1997) de PM indicando que no pueden ser generalizables debido a que el comportamiento de los consumidores es diferente según las latitudes (Bartikowski et al.,2019; Roy, 2021). con respecto al establecimiento de la marca, los detalles si cuentan, es por ello que al momento de la creación de un logotipo las letras en mayúsculas o minúsculas tienen efectos en las actitudes de los consumidores. Mientras que las minúsculas están asociadas con el género femenino, las mayúsculas lo están con el masculino Wen \& Lurie (2018).

Los estudios también se han extendido al ámbito deportivo (Hohenberger \& Grohs, 2020). Se hace investigaciones de PM para fines de PE desde la teoría de la transferencia de los rasgos, lo cual cuenta de tres pasos los rasgos se activan durante la interpretación de los comportamientos descritos, esos rasgos activados se asocian con las personas presentes durante el Paso 1, las asociaciones creadas por el Paso 2 implícitamente en influir en las impresiones de rasgos de esas personas (Bikash et al., 2021).

La preocupación por investigar la PM para el PE también se extiende al sector educativo, con esta nueva modalidad de clases virtuales a causa de la pandemia COVID 19, la interacción entre estudiantes (clientes) en entornos virtuales genera comportamientos y actitudes relacionados con la marca (Perera et al.,2020; Woldearegay, 2021). En ese mismo orden de ideas, la motivación, singularidad de la marca, relaciones interpersonales, innovación y satisfacción de los estudiantes, se convierten en factores determinantes en la supervivencia de las instituciones educativas Brzaković et al.,2019; Lee et al., 2018). 


\section{Figura 3}

Contribución de acuerdo al sector económico

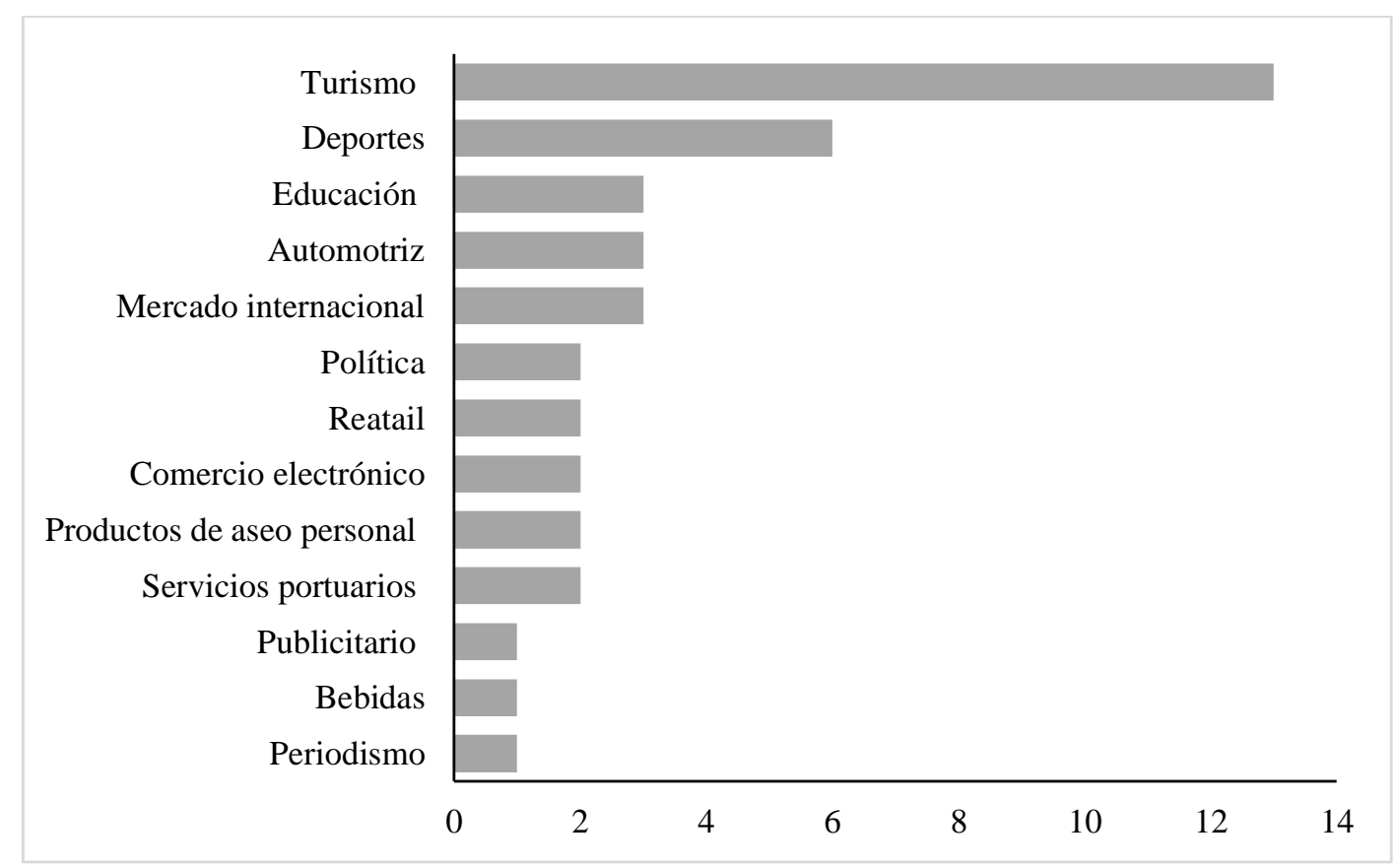

Fuente: elaboración propia.

\section{Modelos de personalidad de marca para fines de posicionamiento}

Durante estos tres últimos años han surgido algunos modelos de PM que permiten tener una mejor posición empresarial, las cueles se muestran en la Figura 4.

Modelos BCP. Nguyen et al. (2018) propusieron un modelo de coherencia de la cartera de marca (BPC), que permite conocer mejor a los consumidores, este constructo está compuesto por coherencia de diseño, de personalidad y de estado. Ofrece herramientas útiles que permiten una mejor gestión en las empresas. 


\section{Figura 4}

Modelos de personalidad de marca

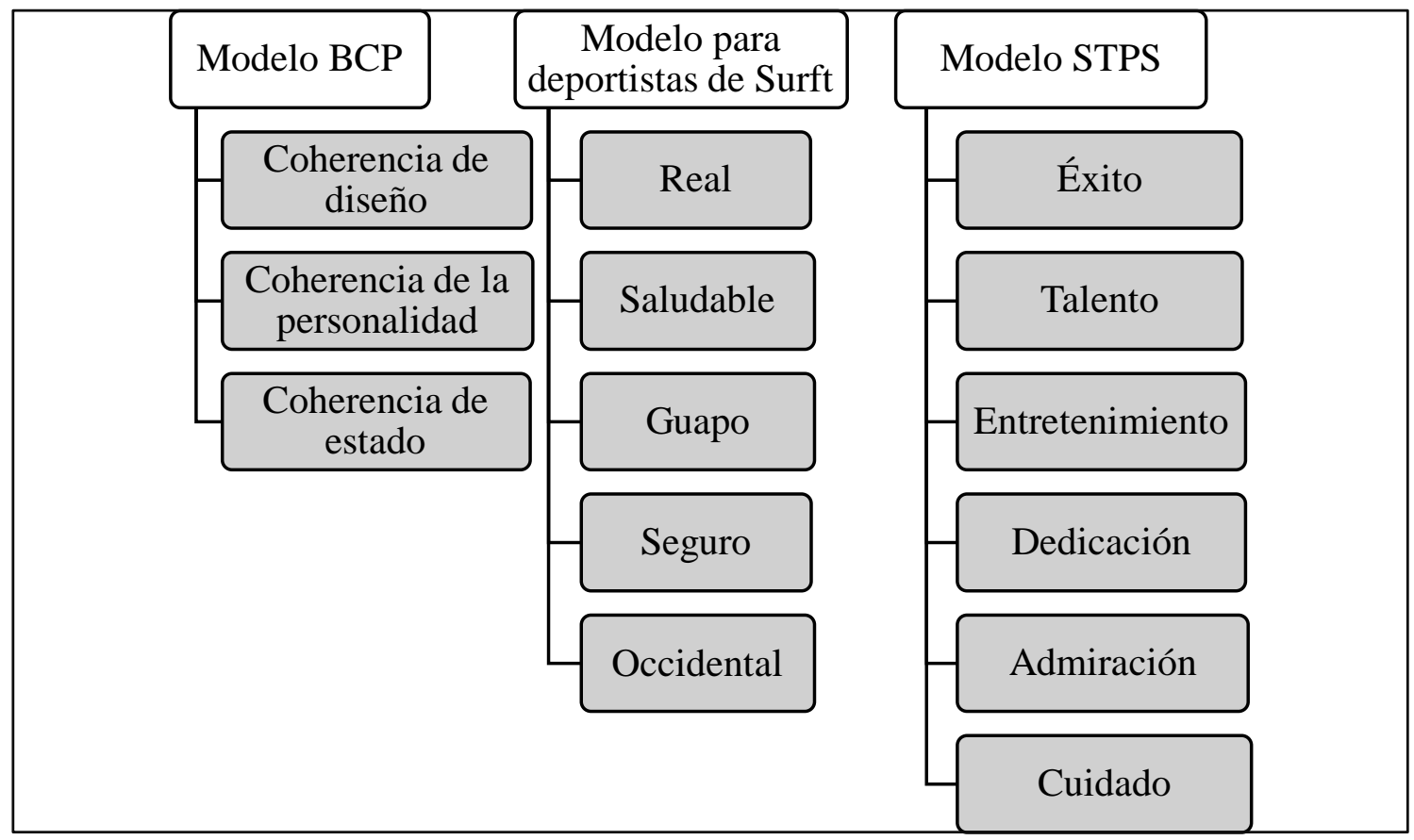

Fuente: elaboración propia.

Kakitek (2018) propone un modelo de PM a partir del modelo Aaker (1997), su propósito fue construir una escala ultracorta por motivos prácticos ya que considera que la PM es solo una de varias medidas en un constructo. Finalmente, el constructo quedó establecido por cinco dimensiones (real, saludable, guapo, seguro, occidental) y veintitrés ítems que miden la PM del surfista.

Modelo para deportistas de Surf. Para este modelo, Stadler et al. (2018) enfatizan la necesidad de medir la PM de los equipos deportivos, en ese sentido su investigación consistió en desarrollar un modelo que se divide en seis factores (éxito, talento, entretenimiento, dedicación, admiración y cuidado) estos factores divididos en 18 ítems que miden la variable antes mencionada. Sus resultados muestran que los equipos deportivos se concentran en el factor desempeño, carácter, este último es el que más identifica a los equipos deportivos.

Modelo STPS. 


\section{Discusión}

El estudio de la PM inició en el año (Ogilvy, 1955), este ha sido estudiado por muchos autores desde diferentes perspectivas, sectores, técnicas y modelos. Sin embargo, para 2002 solo Merrilees \& Miller (2002) habían estudiado la PM en el sector Retail, desde esa fecha a 2021 se han encontrado solo 5 estudios en este sector, mientras en el sector turismo se muestra mayor profundidad en el estudio (Kim et al., 2018; Stancu et al., 2021; Wen \& Lurie, 2018; Zentes et al., 2008), y se ha ido poco a poco extendiendo a otros sectores económicos.

A partir de las investigaciones previas es necesario sentar las bases para nuevas investigaciones y contribuciones relativas a la PM, que consideren diferentes maneras de medirla como lo hizo Moussa (2021), quien afirma que se puede usar emojis en lugar de palabras para medir la PM de forma universal, así también Sagar et al., (2011) presentan un Índice de Posicionamiento Ético (EPI), que es una escala de ranking innovadora basada en las cuestiones éticas en el posicionamiento de la marca, su trabajo se basa en idea central de que la ética se puede utilizar como un diferenciador de producto y puede crear una ventaja estratégica, dicho índice está constituido por cinco elementos básicos del posicionamiento de marca: identidad de marca, marca la imagen, la PM, el conocimiento de la marca y la comunicación de la marca, y se combinan con tres elementos básicos de la ética; creencias, valores, símbolos y costumbres. Mientras que Westjohn et al., (2016) encontraron una relación positiva entre la orientación del consumo global y su preferencia por el posicionamiento global de la marca. Por su parte, la investigación de Rasooli et al., (2012), señala que es necesario crear una distinción en comparación con los competidores para crear conocimiento de marca y asociación de marca. Además, Gammon et al., (2011) encontraron que el posicionamiento global de la marca (comparado con posicionamiento de marca local) mejora las evaluaciones de marca, especialmente para sujetos con niveles más altos de creencia en la ciudadanía global.

Adicionalmente, se deben considerar los estudios desde las diferentes técnicas para el PE. En esta línea Taecharungroj (2018) propone el cuadro de divergencia que tiene el propósito de ayudar en la mejora de la gestión administrativa de las marcas a fin de desarrollar la estrategia adecuada del $\mathrm{PE}$, que se compone de varios niveles y evalúan los atributos y la PM con sus sub marcas o marcas 
paraguas y otra técnica es el mapa perceptual que ayuda a los gerentes al posicionamiento de marca. (Rasooli et al., 2018). Cabe mencionar que el estudio de la PM en los últimos años se ha profundizado y el presente trabajo da cuenta de que es posible abordarlo desde múltiples enfoques.

\section{Conclusiones}

Las principales herramientas de posicionamiento desde la teoría de la PM, permiten analizar el posicionamiento estratégico de una marca, las más adecuadas en su aplicación son; el análisis de correspondencia (Hoare \& Bock, 2019; Baştuğ et al., 2019; Rutter et al., 2019; Rutter et al., 2018) y el mapeo perceptual (Rasooli et al.,2018).

Las principales contribuciones de PM para fines de PE de acuerdo al sector económico en los últimos cuatro años son; es en el turismo, deporte, educación, automotriz, mercado internacional, política, retail, comercio electrónico, productos de aseo personal, servicios portuarios, publicidad, bebidas y periodismo.

Por otra parte, los modelos de PE desde la teoría de la PM surgidos en estos últimos cuatro años son; El modelo de coherencia de la cartera de marca (BCP) (Nguyen et al., 2018), el modelo para deportistas (Kakitek, 2018) y el modelo STPS (Stadler et al.,2018) y aunque todos evalúan la PM la miso tiempo se componen de atributos distintos. Aunado a esto, a raíz del avance tecnológico y la virtualización, la minería de textos es considerado como una herramienta muy potente que se utiliza para medir la PM para fines de PE (Ruei et al., 2019).

En estos últimos años, las investigaciones han estado enfocados en analizar la PM. Sin embargo, habido muy poca preocupación por analizar la personalidad del consumidor y como esta es congruente o no con la PM. Además, si bien es cierto que los estudios de la PM han buscado medir el constructo con los modelos generados, sería adecuado ampliar la ventana de observación e incluir en las investigaciones variables como imagen de marca, lealtad y el comportamiento del consumidor usando diferentes técnicas, minería de datos, emojis, índices entre otras.

Finalmente, algunos esfuerzos (Aichner et al., 2016; Laroche et al., 2003; Strizhakova \& Coulter, 2019) sugieren que la investigación sobre los productos extranjeros puede variar entre subculturas 
dentro de un país, por lo que se alienta a investigar la efectividad de las estrategias de posicionamiento de marca en subculturas y un estudio reciente de Moussa (2021), aporta que mediante representaciones pictográficas de emociones, ideas, reacciones y pensamientos se puede contribuir claramente a la medición intercultural eficaz de la PM, y también es posible enfocarlo al PE. Así entonces, el estudio de la PM con fines de PE ha ido avanzando e innovando principalmente en los últimos 4 años.

\section{Referencias}

(1) Aaker, D. (2020). Winning against a dominant brand. Journal of Brand Strategy, 9(2), 103112. https://hstalks.com/article/5762/winning-against-a-dominant-brand/

(2) Aaker, D. A. (1996). Criando e administrando marcas de sucesso (Futura (Ed.)). Futura.

(3) Aaker, J. L. (1997). Dimensions of brand personality. Journal of Marketing Research, 34(3), 347-356. https://doi.org/10.2307/3151897

(4) Aaker, J. L., Benet-Martínez, V. \& Garolera, J. (2001). Consumption symbols as carriers of culture: A study of Japanese and Spanish brand personality constructs. Journal of Personality and Social Psychology, 81(3), 492-508. https://doi.org/10.1037/0022-3514.81.3.492

(5) Aichner, T., Coletti, P., Forza, C., Perkmann, U. \& Trentin, A. (2016). Effects of Subcultural Differences on Country and Product Evaluations: A Replication Study. Journal of Global Marketing, 29(3), 115-127. https://doi.org/10.1080/08911762.2015.1138012

(6) Alcaniz, J. E. B. y López, N. (2000). Estrategia de empresa y posicionamiento de producto en la industria del automóvil: un análisis comparado. Economía Industrial, (332), 29-42. https://www.mincotur.gob.es/Publicaciones/Publicacionesperiodicas/EconomiaIndustrial/Rev istaEconomiaIndustrial/332/07.J.\%20E.BIGNE.pdf

(7) American Marketing Association. (2019). Common Language in Marketing. https://www.ama.org/topics/branding/

(8) American Marketing Association. (2021). Brand Personality. Marketer Academy Measurement. https://marketing-dictionary.org/b/brand-personality/

(9) Bartikowski, B., Fastoso, F. \& Gierl, H. (2019). Luxury cars Made-in-China: Consequences for brand positioning. Journal of Business Research, 102(May 2018), 288-297. https://doi.org/10.1016/j.jbusres.2019.01.072 
(10) Baştuğ, S., Şakar, G. D. \& Gülmez, S. (2019). An application of brand personality dimensions to container ports: A place branding perspective. Journal of Transport Geography, August. 82. https://doi.org/10.1016/j.jtrangeo.2019.102552

(11) Bennett, R. (1997). Communicator credibility, personality factors and customer responses to comparative advertising claims. Marketing Intelligence \& Planning, 15(2), 85-96. https://doi.org/10.1108/02634509710165885

(12) Bikash, K. R., Gochhayat, J. \& Samanta, S. (2021). Revisiting brand personality attributes: Mediating role of brand attitude. International Journal of Asian Business and Information Management, 12(2), 124-136. https://doi.org/10.4018/IJABIM.20210401.oa8

(13) Brzaković, A., Brzaković, T. \& Brzaković, P. (2019). The determinants of brand positioning in higher education - what dominantly influences students' satisfaction? Croatian Journal of Education, 21(2), 407-436. https://doi.org/10.15516/cje.v21i2.3136

(14) Capelli, S. \& Jolibert, A. (2009). Metaphor's validity in marketing research. Psychology and Marketing, 26(12), 1079-1090. https://doi.org/10.1002/mar.20313

(15) Caprara, G. V., Barbaranelli, C. \& Guido, G. (2001). Brand personality: how to make the metaphor fit? Journal of Economic Psychology, 22(3), 377-395. https://doi.org/10.1016/S01674870(01)00039-3

(16) Caruana, A., Berthon, P., Pitt, L.F. \& Berthon, J. P. (2007). Psychometric properties of the Brand personality scale: evidence from a business school. Psychological Reports, 100(3), 789-794. https://doi.org/10.2466/pr0.100.3.789-794

(17) Chacko, P. S., Ramanathan, H. N. \& Prashar, S. (2019). Desire and likeliness to buy luxury products: mapping perceptions using multi-dimensional scaling. International Journal of Indian Culture and Business Management, 18(2), 123. https://doi.org/10.1504/ijicbm.2019.10019340

(18) Chicaíza, L. A., Riaño, M. I., Rojas, S. P. y Garzón, C. (2017). Revisión sistemática de la literatura en administración. Documentos FCE-CID Escuela de Administración y Contaduría Pública, 29, 1-18. http://www.fce.unal.edu.co/publicaciones/images/doc/documentosadministracion-29.pdf

(19) Choudhury, M., Mishra, B. B. \& Mohanty, P. K. (2019). Perceptual Mapping of SED and Brand Choice to Purchase Car. Pacific Business Review International, 12(1), 94-107. https://ssrn.com/abstract=3805193

(20) Colmenares, O. y Saavedra, J. (2008). Dimension of the Brand personality. Case study: 
Pharmacy chains. Revista Venezolana de Gerencia, 13(42), 220-232. https://produccioncientificaluz.org/index.php/rvg/article/view/10494/10482

(21) Deb, M. (2020). An empirical investigation on heritage destination positioning and loyalty. Current Issues in Tourism, 20(1), 1-14. https://doi.org/10.1080/13683500.2020.1803806

(22) Ekinci, Y. \& Hosany, S. (2006). Destination personality: An application of brand personality to tourism destinations. Journal of Travel Research, 45(2), 127-139. https://doi.org/10.1177/0047287506291603

(23) Escobar, M., Cardoza, C., Vega, J. \& Cañas, M. (2017). Propuesta de modelo: personalidad de marca en cadenas de farmacias en Chile. Suma de Negocios, 8(17), 47-56. https://doi.org/10.1016/j.sumneg.2016.08.001

(24) Espinoza, M. A. \& Gallegos, D. D. (2019). Benchmarking, how and from where?: A systematic $\begin{array}{lllll}\text { review of } & \text { literature. } & \text { Espacios, } & 40(37), & \end{array}$ https://www.revistaespacios.com/a19v40n37/a19v40n37p16.pdf

(25) Fournier, S. (1998). Consumers and Their Brands: Developing Relationship Theory in Consumer Research. Journal of Consumer Research, 24(4), 343-373. https://econpapers.repec.org/RePEc:oup:jconrs:v:24:y:1998:i:4:p:343-73

(26) Foxall, G. R. \& Haskins, C. G. (1986). Cognitive Style and Consumer Innovativeness: An Empirical Test of Kirton's Adaption-Innovation Theory in Food Purchasing. Marketing Intelligence \& Planning, 4(1), 26-46. https://doi.org/10.1108/eb045723

(27) Gammoh, B. S., Koh, A. C. \& Okoroafo, S. C. (2011). Consumer culture brand positioning strategies: an experimental investigation. Journal of Product \& Brand Management, 20(1), 4857. https://doi.org/10.1108/10610421111108012

(28) Garanti, Z. \& Kissi, P. S. (2019). The effects of social media brand personality on brand loyalty in the Latvian banking industry: The mediating role of brand equity. International Journal of Bank Marketing, 37(6), 1480-1503. https://doi.org/10.1108/IJBM-09-2018-0257

(29) Gita, I. E., Beca, M. T. \& Cetina, I. (2018). Brand name traslation as a decisive factor in positionning foreign brands on Chinese market (II). Transilvania, 53(4), 57-63. http://publications.lib.chalmers.se/records/fulltext/245180/245180.pdf\%0Ahttps://hdl.handle.n et/20.500.12380/245180\%0Ahttp://dx.doi.org/10.1016/j.jsames.2011.03.003\%0Ahttps://doi.or g/10.1016/j.gr.2017.08.001\%0Ahttp://dx.doi.org/10.1016/j.precamres.2014.12

(30) Glińska, E. \& Rudolf, W. (2019). City brand personality projected by municipalities from 
Central and Eastern Europe countries-A comparison of Facebook usage. Sustainability, 11(19), 5440. https://doi.org/10.3390/su11195440

(31) Gouvêa, M. A. (1991). Uma contribuição para o desenvolvimento de estratégias de posicionamento no contexto de marketing bancário. Universidade de São Paulo.

(32) Habibi, A., Ariffin, A. A. M. \& Aziz, N. A. (2018). The influence of perceived benefits, perceived sacrifices and perceived value on behavioural intention in the context of medical tourism. International Journal of Services, Economics and Management, 9(3-4), 295-316. https://doi.org/10.1504/IJSEM.2018.097789

(33) Hanna, S. \& Rowley, J. (2019). The projected destination brand personalities of European capital cities and their positioning. Journal of Marketing Management, 35(11-12), 1135-1158. https://doi.org/10.1080/0267257X.2019.1647274

(34) Hoare, J. \& Bock, T. (2019). A brand's eye view of correspondence analysis. International Journal of Market Research, 61(1), 12-21. https://doi.org/10.1177/1470785318801480

(35) Hohenberger, C. \& Grohs, R. (2020). Old and exciting? Sport sponsorship effects on brand age and brand personality. Sport Management Review, 23(3), 469-481. https://doi.org/10.1016/j.smr.2019.05.002

(36) Hooley, G. J. y Saunders, J. (1996). Posicionamento competitivo. Makron Books.

(37) Hosany, S., Ekinci, Y. \& Uysal, M. (2006). Destination image and destination personality: An application of branding theories to tourism places. Journal of Business Research, 59(5), 638642. https://doi.org/10.1016/j.jbusres.2006.01.001

(38) Iacobucci, D. \& Grisaffe, D. (2018). Perceptual maps via enhanced correspondence analysis: representing confidence regions to clarify brand positions. Journal of Marketing Analytics, 6(3), 72-83. https://doi.org/10.1057/s41270-018-0037-7

(39) Jaksic, A., Jankovic, M. \& Seric, N. (2019). Montenegro as High-Quality Sports Tourism Destination- Trends and Perspectives. Sport Mont, 17(1), 93-95. https://doi.org/10.26773/smj.190218

(40) Kakitek, A. (2018). Application of Aaker's brand personality scale on human brands in surf sports. Journal of Management and Business Administration. 26(4), 11-31. https://doi.org/10.7206/jmba.ce.2450-7814.240

(41) Kapferer, J. N. (1992). Strategic brand management: New approaches to creating and evaluating brand equity. The Journal of Marketing, 58(3), 118-119 https://doi.org/10.2307/1252315 
(42) Kim, H. S. (2000). Examination of brand personality and brand attitude within the apparel product category. Journal of Fashion Marketing and Management, 4(3), 243-252. https://doi.org/10.1108/eb022593

(43) Kim, P., Vaidyanathan, R., Chang, H. \& Stoel, L. (2018). Using brand alliances with artists to expand retail brand personality. Journal of Business Research, 85(2), 424-433. https://doi.org/10.1016/j.jbusres.2017.10.020

(44) Kotler, P. \& Keller, K. (2012). Dirección de Marketing (Pearson (Ed.)). Pearson Education.

(45) Kotler, P. (2000). Administração de Marketing (4a ed). Atlas.

(46) Kotler, P., Kartajaya, H. \& Setiawan, I. (2017). Marketing 4.0. John Wiley \& Sons (Ed.); (Primera ed). https://www.nima.today/wp-content/uploads/2018/11/Marketing-4.0-PhilipKotler-Hermawan-Kartajaya-And-Iwan-Setiawan.pdf

(47) Lara, J. S., Rojas, C. \& Duque, E. J. (2019). Discovering emerging research topics for brand personality: A bibliometric analysis. Australasian Marketing Journal, 27(4), 1-12. https://doi.org/10.1016/j.ausmj.2019.06.002

(48) Laroche, M., Papadopoulos, N., Heslop, L. \& Bergeron, J. (2003). Effects of subcultural differences on country and product evaluations. Journal of Consumer Behaviour, 2(3), 232-247. https://doi.org/10.1002/cb.104

(49) Lee, S. \& Kim, D. Y. (2018). Brand personality of Airbnb: application of user involvement and gender differences. Journal of Travel and Tourism Marketing, 35(1), 32-45. https://doi.org/10.1080/10548408.2017.1284030

(50) Liu, Z., Huang, S. \& Liang, S. (2019a). Does Brand Personification Matter in Consuming Tourism Real Estate Products? A Perspective on Brand Personality, Self-congruity and Brand Loyalty. Journal of China Tourism Research, 15(4), 435-454. https://doi.org/10.1080/19388160.2018.1516586

(51) Liu, Z., Huang, S. \& Liang, S. (2019b). Does Brand Personification Matter in Consuming Tourism Real Estate Products? A Perspective on Brand Personality, Self-congruity and Brand Loyalty. Journal of China Tourism Research, 15(4), 435-454. https://doi.org/10.1080/19388160.2018.1516586

(52) Llanos, G. R. \& Merigo, J. M. (2019). Overview of brand personality research with bibliometric indicators. Kybernetes, 48(3), 546-569. https://doi.org/10.1108/K-02-2018-0051

(53) López, A., Muñoz, L. \& Casero, A. (2020). Strategies in journalistic branding on social media: 
The influence of public and business dimensions according to future journalists' perceptions. Communication Today, $\quad$ 11(1), 54-68. http://repositori.uji.es/xmlui/handle/10234/187624https://www.communicationtoday.sk/strateg ies-in-journalistic-branding-on-social-media-the-influence-of-public-and-business-dimensionsaccording-to-future-journalists-perceptions/

(54) Magnusson, P., Westjohn, S. A. \& Sirianni, N. J. (2019). Beyond country image favorability: How brand positioning via country personality stereotypes enhances brand evaluations. Journal of International Business Studies, 50(3), 318-338. https://doi.org/10.1057/s41267-018-0175-3

(55) Merrilees, B. \& Miller, D. (2002). Antecedents of brand-personality in Australian retailing: an exploratory study. Proceedings of the ANZMAC Conference 2002.

(56) Moussa, S. (2021). Measuring brand personality using emoji: findings from Mokken scaling. Journal of Brand Management, 28(2), 116-132. https://doi.org/10.1057/s41262-020-00220-8

(57) Murase, H. \& Bojanic, D. (2004). An examination of the differences in restaurant brand personality across cultures. Journal of Hospitality and Leisure Marketing, 11(2-3), 97-113. https://doi.org/10.1300/J150v11n02_08

(58) Nazim, S. \& Rajeswari, M. (2019). Impact of underdog narrative advertising strategy on brand positioning and customer purchase intentions. International Journal of Recent Technology and Engineering, 8(3), 3208-3213. https://doi.org/10.35940/ijrte.C4667.098319

(59) Nguyen, H. T., Zhang, Y. \& Calantone, R. J. (2018). Brand portfolio coherence: Scale development and empirical demonstration. International Journal of Research in Marketing, 35(1), 60-80. https://doi.org/10.1016/j.ijresmar.2017.11.003

(60) Ogilvy, D. (1955). The image of the brand-a new approach to creative operations. Reprinted by Courtesy of Ogilvy \& Mather, 1-6. https://www.markenlexikon.com/texte/ogilvy_the-image-ofthe-brand_1955.pdf

(61) Perera, C. H., Nayak, R. \& Nguyen, L. T. Van. (2020). Social brand engagement and brand positioning for higher educational institutions: an empirical study in Sri Lanka. Journal of Marketing for Higher Education, O(0), 1-18. https://doi.org/10.1080/08841241.2020.1841068

(62) Porter, M. (1989). Vantagem competitiva (16a ed).

(63) Porter, M. E. (2016). Ventaja Competitiva (Grupo Editorial Patria (Ed.)). Grupo Editorial Patria.

(64) Quintal, V. A., Lwin, M., Phau, I. \& Lee, S. (2019). Personality attributes of botanic parks and their effects on visitor attitude and behavioural intentions. Journal of Vacation Marketing, 25(2), 
176-192. https://doi.org/10.1177/1356766718760089

(65) Radler, V. M. (2018). 20 Years of brand personality: A bibliometric review and research agenda. Journal of Brand Management, 25(4), 370-383. https://doi.org/10.1057/s41262-017-0083-z

(66) Rai, S. \& Jogendra, K. N. (2018). Role of event personality and exhibitors' eudaimonic and hedonic happiness in predicting event advocacy intentions: an empirical study. International Journal of Event and Festival Management, 9(1), 86-103. https://doi.org/https://doi.org/10.1108/IJEFM-09-2017-0056

(67) Rasooli, M., Talebpour, M., Javadipour, M. \& Ghodsi, P. (2012). The model of industry and university association in the field of sport. The first international conference on privatization and investment in sport. Tehran.

(68) Rasooli, S. M., Bkhsheshi, M. F., \& Zareian, H. (2018). Brand positioning of the Sport Sciences Research Institution of Iran using Perceptual Mapping Technique. Annals of Applied Sport Science, 6(1), 103-113. https://doi.org/10.29252/aassjournal.6.1.103

(69) Ries, A. \& Trout, J. (2002). Posicionamento: a batalha por sua mente (20a ed). Makron Books.

(70) Rojas, J. I., Erenchun, I. \& Silva, E. (2004). The Ford Brand Personality in Chile. Corporate Reputation Review, 7(3), 232-251. https://doi.org/10.1057/palgrave.crr.1540223

(71) Rojas, J. I., Hine, M. J. \& Rod, M. (2018). Brand Personalities of Global Wine Exporters: A Collective Reputation Theory Perspective. Journal of International Food and Agribusiness Marketing, 30(1), 88-105. https://doi.org/10.1080/08974438.2017.1387884

(72) Roy, S. (2021). Cross-Cultural Dissimilarities in the Perception of Brand Personality of Select Smart phones : Evidence from West Bengal, India and Bangladesh Keywords. XXIX(2), 25-52. https://management-review.nmims.edu/wp-content/uploads/2021/06/Article-3.pdf

(73) Ruei. L., Hsiu, T., Koong, L., Yu, C. \& Cheng, T. (2019). Sentiment analysis of brand personality positioning through text mining. Journal of Information Technology Research, 12(3), 93-103. https://doi.org/10.4018/JITR.2019070106

(74) Rutter, R. N., Hanretty, C. \& Lettice, F. (2018a). Political Brands: Can Parties Be Distinguished by Their Online Brand Personality? Journal of Political Marketing, 17(3), 193-212. https://doi.org/10.1080/15377857.2015.1022631

(75) Rutter, R., Nadeau, J., Aagerup, U. \& Lettice, F. (2019). The Olympic Games and associative sponsorship: Brand personality identity creation, communication and congruence. Internet Research, 30(1), 85-107. https://doi.org/10.1108/INTR-07-2018-0324 
(76) Rutter, R., Nadeau, J., Lettice, F., Lim, M. \& Shamaisi, S. Al. (2018). Place branding of seaports in the Middle East. Place Branding and Public Diplomacy, 14(3), 197-212. https://doi.org/10.1057/s41254-017-0072-8

(77) Sagar, M., Khandelwal, R., Mittal, A. \& Singh, D. (2011). Ethical Positioning Index (EPI): An innovative tool for differential brand positioning. Corporate Communications, 16(2), 124-138. https://doi.org/10.1108/13563281111141651

(78) Schlansker, J. T. (1946). The Journal of Psychology: Interdisciplinary and Applied Maternal Sensitivity : Vocalization During Infant Feedings. The Journal of Psychology Interdisciplinary and Applied, 21(1), 37-41. https://doi.org/http://dx.doi.org/10.1080/00223980.1946.9917275

(79) Shank, M. D. \& Langmeyer, L. (1994). Does personality influence brand image? Journal of Psychology: Interdisciplinary and Applied, 128(2), 157-164. https://doi.org/10.1080/00223980.1994.9712719

(80) Siguaw, J. A., Mattila, A. \& Austin, J. R. (1999). The Brand-personality scale. Cornell Hotel and Restaurant Administration Quarterly, 40(3), 48-5. https://doi.org/10.1016/s00108804(99)80037-8

(81) Stadler Blank, A., Koenigstorfer, J. \& Baumgartner, H. (2018a). Sport team personality: It's not all about winning! Sport Management Review, 21(2), 114-132. https://doi.org/10.1016/j.smr.2017.05.004

(82) Stadler Blank, A., Koenigstorfer, J. \& Baumgartner, H. (2018b). Sport team personality: It's not all about winning! Sport Management Review, 21(2), 114-132. https://doi.org/10.1016/j.smr.2017.05.004

(83) Stancu, S., Constantin, A. M., Cristea, A. \& Cristea, N. (2021). Modelling the interdependences between brand logo design and consumers' perceptions regarding brand personality dimensions. Economic Computation and Economic Cybernetics Studies and Research, 55(1), 269-283. https://doi.org/10.24818/18423264/55.1.21.17

(84) Steenkamp, J., Trijp, H. \& Berge, T. (1994). Perceptual Mapping Based on Idiosyncratic Sets of Attributes. Journal of Marketing Research, 31(1), 15. https://doi.org/10.2307/3151943

(85) Strizhakova, Y. \& Coulter, R. (2019). Consumer cultural identity: local and global cultural identities and measurement implications. International Marketing Review, 36(5), 610-627. https://doi.org/10.1108/IMR-11-2018-0320

(86) Su, N. \& Reynolds, D. (2019). Categorical differences of hotel brand personality: Identifying 
competition across hotel categories. International Journal of Contemporary Hospitality Management, 31(4), 1801-1818. https://doi.org/10.1108/IJCHM-05-2018-0354

(87) Taecharungroj, V. (2018). City-district divergence grid: A multi-level city brand positioning tool. Place Branding and Public Diplomacy, 14(2), 101-114. https://doi.org/10.1057/s41254017-0077-3

(88) Serralvo, F. y Tadeu, F. (2005). Tipologías del posicionamiento de marcas. Un estudio conceptual en Brasil y en España. Revista Galega de Economía, 14(1-2), 1-16. https://www.redalyc.org/pdf/391/39114226.pdf

(89) Van, M. (1997). Brand strategy needs turning back to front. Marketing Intelligence \& Planning, 15(3), 157-159. https://doi.org/10.1108/02634509710165966

(90) Vinyals, S. \& Mohammadi, L. (2018). City brand projected personality: A new measure to assess the consistency of projected personality across messages. Communication and Society, 31(4), 91-108. https://doi.org/10.15581/003.31.4.91-108

(91) Vriens, M., Chen, S. \& Vidden, C. (2019). Mapping brand similarities: Comparing consumer online comments versus survey data. International Journal of Market Research, 61(2), 130-139. https://doi.org/10.1177/1470785318810106

(92) Wen, N. \& Lurie, N. H. (2018). The Case for Compatibility: Product Attitudes and Purchase Intentions for Upper versus Lowercase Brand Names. Journal of Retailing, 94(4), 393-407. https://doi.org/10.1016/j.jretai.2018.10.002

(93) Westjohn, S. A., Arnold, M. J., Magnusson, P. \& Reynolds, K. (2016). The Influence of Regulatory Focus on Global Consumption Orientation and Preference for Global versus Local Consumer Culture Positioning. Journal of International Marketing, 24(2), 22-39. https://doi.org/10.1509/jim.15.0006

(94) Woldearegay, A. G. (2021). Reputation of Addis Ababa University in the Eyes of Students: A College-Level Perspective from Teacher Preparation Programs. Education Research International, 2021. https://doi.org/10.1155/2021/5514195

(95) Won, E., Oh, Y. \& Choeh, J. (2018). Perceptual mapping based on web search queries and consumer forum comments. International Journal of Market Research, 60(4), 394-407. https://doi.org/10.1177/1470785317745971

(96) Zentes, J., Morschett, D. \& Schramm-Klein, H. (2008). Brand personality of retailers - An analysis of its applicability and its effect on store loyalty. International Review of Retail, 
Distribution and Consumer Research, 187-184. https://doi.org/10.1080/09593960701868282

Cómo citar este artículo: Cruz, J., Haro, K. y Soria, J. (2021). Revisión sistemática: situación actual de la personalidad de marca para el posicionamiento estratégico. Tendencias, 23(1), 315340. https://doi.org/10.22267/rtend.222301.192 